Revista científica, arbitrada e indizada, bajo la modalidad electrónica.

Recibido: $27 / 10 / 2019$

Aceptado: 05/11/2019

\title{
La violencia como fenómeno transnacional articulado al crimen organizado
}

Violence as a transnational phenomenon articulated to organized crime

Víctor Romero

Vr003,qhse@gmail.com

Luis Moya

luismoyave@gmail.com

Luis Mantilla

mantillacala@gmail.com

José Ramón Blanco

fireschool2@gmail.com

Universidad Yacambú

Venezuela

\section{RESUMEN}

Este trabajo tiene como propósito analizar la violencia como fenómeno transnacional articulado al crimen organizado. Por lo tanto, la investigación que se expone encuadrada de acuerdo a su naturaleza en un enfoque postpositivista, cualitativo, tipo documental sociojurídica. Asimismo, utiliza un diseño multi-métodos, basándose en el estudio interpretativo-crítico, el cual corresponde a trabajos fundamentados en la revisión exhaustiva de documentación. De igual forma, basa su desarrollo en un diseño bibliográfico. Arrojando como resultado que en los cuatro países examinados, la violencia generada producto de factores económicos, políticos y sociales, así como la ingobernabilidad en estos Estados, se encuentra vinculada directamente con la generación del crimen organizado, ya que es evidente su afianzamiento en todas las modalidades, trayendo consigo deterioro de las instituciones públicas garantizadoras del bienestar social, debido a la impunidad ante las acciones delictivas. Al igual que en otros países del mundo, este flagelo no solo afecta la institucionalidad del Estado al hacerlo ingobernable, sino la seguridad de las personas, quienes se sienten constantemente amenazadas en su integridad física. Adicionalmente, estimula la corrupción, favoreciendo otras acciones delictivas como el secuestro y la extorsión. Concluyendo que Colombia, Brasil, Guatemala y Venezuela comparten ciertos indicadores facilitadores de la presencia de actividades delictivas fomentadas por el crimen organizado, las cuales afectan de manera significativa no sólo a las poblaciones de estos países, sino también a aquellos estados por donde estos grupos transitan para realizar sus transacciones.

Palabras Clave: Violencia, Crimen organizado, Impunidad, Actividades delictivas. 


\title{
Revista científica, arbitrada e indizada, bajo la modalidad electrónica.
}

\begin{abstract}
The purpose of this work is to analyze violence as a transnational phenomenon linked to organized crime. Therefore, the research presented is framed according to its nature in a postpositive, qualitative, socio-legal documentary type approach. Likewise, it uses a multimethod design, based on the interpretative-critical study, which corresponds to works based on the exhaustive review of documentation. Similarly, it bases its development on a bibliographic design. Throwing as a result that in the four countries examined, the violence generated as a result of economic, political and social factors, as well as the ungovernability in these States, is directly linked to the generation of organized crime, since its consolidation is evident in all modalities, bringing with it the deterioration of public institutions that guarantee social welfare, due to impunity for criminal actions. As in other countries of the world, this scourge affects not only the institutionality of the State by making it ungovernable, but the security of people, who feel constantly threatened in their physical integrity. Additionally, it encourages corruption, favoring other criminal actions such as kidnapping and extortion. Concluding that Colombia, Brazil, Guatemala and Venezuela share certain facilitating indicators of the presence of criminal activities promoted by organized crime, which significantly affect not only the populations of these countries, but also those states through which these groups transit to carry out your transactions.
\end{abstract}

Key words: Violence, Organized crime, Impunity, Criminal activities.

\section{INTRODUCCIÓN}

Partiendo de lo establecido en la legislación penal venezolana, concretamente en el Código Penal Venezolano (CPV), sobre el significado de Violencia, se precisa que este término no aparece expresamente señalado, sin embargo es descrito a lo largo del texto legal, en sus contenidos normativos sobre delitos o daños a las personas, de igual forma, es considerado en leyes especiales en las cuales se cubre la necesidad sancionatoria producto de este delito, además de incluir una serie de medidas cuyo fin es proteger a la víctima en cualquier proceso.

En correspondencia con lo planteado, para Chihaia (2019), el término violencia se configura como un eje que vincula varias categorías vecinas (crimen, dictadura, guerra, memoria, poder, transgresión, trauma), sin embargo, enfocada en esta investigación, es importante ubicarla en el campo socio crítico, a los fines de poder explicar su correspondencia o articulación con el crimen organizado, que en este trabajo se constituye en la segunda categoría en análisis. 


\section{Revista científica, arbitrada e indizada, bajo la modalidad electrónica.}

Por otro lado, con respecto al crimen organizado, Páucar (2016), sustenta que es el proceso permanente, dinámico y evolutivo de actividades ilegales tanto locales como de proyección internacional, ejecutándose por medio de estructuras organizacionales jerárquicas o flexibles, las cuales tienen por objetivos principales la búsqueda de consolidar una posición económica y/o de poder, por medio de diversos mecanismos como la violencia, influencia, la tecnología, entre otros aspectos.

De allí, que al articular ambas categorías se evidencia la relevancia de analizarlas bajo los parámetros de un estudio comparado entre Colombia, Brasil, Guatemala y Venezuela, a los fines de poder develar algunas similitudes y semejanzas que convergen en estos contextos geográficos. En consecuencia, esta investigación tiene como propósito analizar la violencia como fenómeno transnacional articulado al crimen organizado.

\section{MATERIALES Y MÉTODOS}

Utilizando un enfoque postpositivista, paradigma cualitativo, de tipo documental sociojurídico, esgrimiendo el método comparativo, es realizado un análisis de la violencia como fenómeno transnacional articulado al crimen organizado en Colombia, Brasil, Guatemala y Venezuela, países que son examinados a través de los diferentes autores que han desarrollado proyectos similares, así como el marco jurídico establecido en la materia objeto de este análisis.

Asimismo, siguiendo lo establecido en las Normas UNY (2007), es utilizado un diseño de investigación multi-métodos, basándose en el estudio interpretativo-crítico, el cual corresponde a trabajos fundamentados en la revisión exhaustiva de documentación, efectuados para analizar, además de comprender situaciones y realidades sociales. (pág. 14). De igual forma, fundamenta su desarrollo en un diseño bibliográfico.

En este sentido, la investigación se orientó bajo el enfoque postpositivista, eminentemente cualitativo, que según Pelekais et al., (2016), hace posible relatar experiencias de la vida y darles significado por medio de la revisión de acontecimientos, acciones, normas, valores entre otros aspectos importantes. Por ello, desde la representación del objeto de estudio y del sujeto, este método es manejado para entender las experiencias humanas alrededor de 


\section{Revista científica, arbitrada e indizada, bajo la modalidad electrónica.}

las concepciones generadoras de encuentros sobre los hechos cotidianos asociados a una cercana realidad.

Con respecto a la investigación documental señalan Pelekais et al., (2015), demanda como condición necesaria, un tema seleccionado, delimitado, justificado, producto de la documentación o reflexión personal. Asimismo, requiere plantear un marco de referencia preliminar que haga posible orientar la recolección de información, al igual la redacción posterior del informe de la investigación.

Para lograr lo planteado, fue estructurado el universo de material documental objeto de análisis, quedando integrado por información derivada de diversas fuentes, tales como: Revistas científicas, de divulgación; trabajos de grado y tesis doctorales, porque sus resultados han sido revisados cuidadosamente por expertos antes de ser publicados, artículos científicos, libros, reportes e informes de organismos internacionales certificados en el área. Asimismo, leyes, manuales, resoluciones, normativas, reglamentos nacionales e internacionales. Luego se procedió a definir las unidades básicas de donde se recolectaría la información analizada, estructurando el esquema que sería implementado para la búsqueda bibliográfica especificada. (Pelekais et al., 2015).

Las unidades de estudio, quedaron conformadas por todo el material bibliográfico analizado. Lo cual guarda correspondencia con lo expresado por Bastidas (2011, p.54), previamente citado por Pelekais et al., (2015), para quien la unidad es: "el contexto que contiene la información acerca del evento de estudio dentro de un universo de documento". Aseveración corroborada a través de la ejecución del estudio.

\section{ORIENTACIONES TEÓRICAS}

\section{Violencia}

Con respecto a lo descrito sobre la violencia, autores estudiosos del área como González (2013), consideran que:

Debe entenderse como el uso de la fuerza por parte de sujetos, grupos o instituciones aplicada a otros sujetos, grupos o instituciones con el propósito de obtener algún beneficio o ganancia en contra de su voluntad o de su consentimiento autónomo. Esta dinámica, además de modificar, transformar 


\section{Revista científica, arbitrada e indizada, bajo la modalidad electrónica.}

o mantener por la fuerza una serie de condiciones, actividades, procesos o comportamientos, pone de manifiesto, concreta y simbólicamente el poder, las intenciones y convicciones del agente de la violencia (p. 172).

Asimismo, lo entendido por el referido autor como violencia, distingue en ella dos dimensiones: La violencia estructural u objetiva y la violencia subjetiva. En este sentido, la estructural es la utilización de medios coercitivos para la subordinación de una clase por parte de otra, con la finalidad de obtener una ganancia material, proceso que toma forma en una explotación del humano por el humano y, por ende, requiere de la dominación como una condición sine qua non de las relaciones sociales de producción (p. 173).

De igual forma, se encuentran otros doctrinarios que expresan sus argumentos sobre el significado de la violencia; entre los cuales es posible señalar a Carrara (2014), quien conceptualiza la violencia como una fuerza cualquiera que supere y venza o al menos se trabe en lucha con otra fuerza que le ofrezca resistencia.

Por su parte, Bernall (2015), concibe la violencia, como aquella conducta que con el empleo de medios físicos busca vencer un obstáculo siendo ejercicio o aplicación de la fuerza, presupone necesariamente el objeto sobre el cual se ejerce tal fuerza o violencia.

Asimismo, citando a Chiossone (2013), define el término como el empleo de una energía física hacia la persona pasiva, capaz de producir una coacción personal física, absoluta o relativa, ante cualquier situación.

En este punto es importante aclarar que en la legislación penal venezolana, específicamente en el Código Penal Venezolano (CPV), no se encuentra establecida la definición de violencia, sin embargo el término es descrito a lo largo del Código en sus contenidos normativos sobre delitos o daños a las personas, de igual forma es considerado en leyes especiales en las cuales se cubre la necesidad sancionatoria producto de este delito, además de incluir una serie de medidas cuyo fin es proteger a la víctima en cualquier proceso.

\section{Situación actual de la Violencia como fenómeno transnacional}

Para Solis y Cerna (2014), citando a (Mathieu y Niño, 2011), por diferentes y variadas razones, América Latina es una región articulada con la violencia. Cuando no ha sido la violencia política se exterioriza la violencia delictiva común, la cual ensombrece desde 


\section{Revista científica, arbitrada e indizada, bajo la modalidad electrónica.}

siempre a estos países, de manera más reciente, surge la ejercida por el crimen organizado nacional, además la transnacional. Precisamente esta última faceta es la que representa el mayor desafío para los Estados de la región, los cuales en algunos casos han sido captados por estructuras criminales capaces de chantajear a actores políticos, económicos y legales, para conseguir los fines propuestos en la formulación de sus metas.

De acuerdo con Solis y Cerna (2014), citando a Latinobarómetro (2013), no es de extrañar que la principal inquietud de los ciudadanos latinoamericanos sea la inseguridad, incluso por arriba del desempleo. La ciudadanía se siente amenazada, además de acosada por la delincuencia, en especial por sus expresiones más violentas como lo son el homicidio, secuestro, violencia doméstica, asaltos, entre otros elementos igualmente riesgosos.

Por otro lado, haciendo referencia de Colombia, según Capera y Ñañez (2017), su historia de violencia se ha convertido en un tema de gran importancia para la investigación en las ciencias sociales, quedando demostrado las múltiples dimensiones que tiene el conflicto armado, la concentración de la tierra, la lucha bipartidista entre otras características. En este plano, la violencia se asocia a factores como el uso de territorio, el control político del Estado y en particular el alza del precio de café, lo cual produjo que sectores sociales como el campesinado fueran más vulnerables en esos momentos.

Según los citados autores, el escenario actual del postconflicto se convierte en una apuesta para pensar otro tipo de realidad, aquí toma fuerza la noción de construir una cultura distinta donde se pueda reflexionar sobre respuestas concretas a las necesidades estructurales presentadas por la sociedad colombiana. El proyecto de un tipo de paz territorial es la muestra de desconcentrar el poder, reactivar los actores, líderes, representantes y comunidades para que haga uso de su facultad como sujetos - políticos. Lo cual significa que superar el centralismo, la cultura politiquera y la corrupción que tanto azota a los sectores más excluidos de la política nacional.

Visualizando desde otra perspectiva, Basset et al., (2015), refiere que en el caso colombiano, los factores tendentes a preservar espacios de autoritarismos subnacionales no tienen que ver con la persistencia de unas estructuras político-partidarias hegemónicas, sino más bien con la violencia política cruzada de las guerrillas y de las milicias paramilitares, al 


\section{Revista científica, arbitrada e indizada, bajo la modalidad electrónica.}

limitar las posibilidades de expresión de la ciudadanía. Este punto es complementado por lo expresado en el Plan Colombia, en donde se atribuye al narcotráfico un papel primordial en el desarrollo de la violencia y debilitamiento de las instituciones. (Caballero y Pizano, 2014).

Según lo afirma Sampó (2018), la situación de Brasil en términos de violencia se ha complejizado en los últimos años. De acuerdo con el Atlas da violencia (2017), en 3 semanas de 2017 murió más gente a causa de los homicidios dolosos, que victimas globales en atentados terroristas durante los primeros 5 meses del año. Continua explicando, este fue un año bastante particular debido a una serie de rebeliones en distintos complejos penitenciarios, que no sólo dejaron un saldo de más de 140 muertos, sino dan cuenta del quiebre de una alianza entre grupos criminales, los números dejan de manifiesto un sostenido incremento de la violencia.

Adicionalmente, sostiene la citada autora, de acuerdo con los datos presentados por el Consejo Ciudadano para la Seguridad Pública y la Justicia Penal (2017) - una ONG mexicana - para el año 2016, 20 de las 50 ciudades más violentas del mundo son brasileras y 7, se ubican entre los primeros 20 lugares, considerando los homicidios por cada 100 mil habitantes.

En este orden de ideas, Mesa (2018), en una investigación desarrollada sobre el tema, expone que Centroamérica, particularmente los países del llamado "Triángulo Norte" (Guatemala, El Salvador y Honduras) se ven afectados por elevados niveles de violencia de carácter transnacional, la cual tiene un fuerte impacto político, social, económico y supone uno de los más importantes desafíos para la gobernabilidad democrática de esa región, considerada una de las más violentas del planeta, con un número de víctimas muy elevado, superior a los registrados en los años ochenta, cuando varios países centroamericanos sufrían la guerra. Convirtiéndose en una amenaza a la consolidación democrática, así como al desarrollo económico y social de la región.

Con respecto a Venezuela, el Observatorio de violencia de Venezuela (2020), menciona que la crisis económica, política y social aumenta, el país continua siendo el más violento de América Latina al registrar 16.506 asesinatos en 2019, lo cual significa una tasa de 


\section{Revista científica, arbitrada e indizada, bajo la modalidad electrónica.}

homicidios de 60,3 por 100.000 habitantes. Cifra realmente alarmante y demuestra el elevado nivel de violencia presente.

Asimismo refiere el Observatorio, de acuerdo a los datos arrojados de su investigación, que si bien la tasa de homicidios disminuyó cerca del 25 por ciento en 2019, con respecto a 81,4 por 100,000 que se determinó en 2018, la situación continua siendo objeto de preocupación. Dicha reducción puede atribuirse en parte al mayor control territorial ejercido por los grupos del crimen organizado, así como a la migración de delincuentes menores a otros países de la región.

\section{Crimen organizado}

Examinando la segunda categoría de la investigación que se realiza, es posible encontrar al crimen organizado, el cual es conceptualizado según Cabrera (2017, p.47), como aquella agrupación de personas que con ánimo de lucro, llevan a cabo actividades criminales de forma organizada, además de estructurada, a través del empleo no sólo de violencia o corrupción como recursos, sino de otros métodos y técnicas cada vez más sofisticadas, cuyo alcance traspasa fronteras, vulnerando los derechos fundamentales y afectando a las estructuras básicas de los Estados de una forma agravante. Su accionar atenta contra todo el sistema jurídico, legal, económico o social.

De acuerdo a Prado (2013), la criminalidad organizada desde el punto de vista operativo es:

Toda actividad delictiva que ejecuta una organización de estructura jerárquica o flexible, dedicada de manera continua o permanente a la provisión y comercio de bienes, medios o servicios legalmente restringidos, de expendio fiscalizado o de circulación prohibida, los cuales cuentan con una demanda social interna o internacional, potencial o activa, pero siempre en crecimiento. Además estas actividades criminales se reproducen y extienden aplicando una eficiente dinámica funcional de abuso, inserción o gestión de posiciones expectantes o consolidadas de poder político, económico o tecnológico. (p.60).

Asimismo, el citado autor identifica las siguientes características en la criminalidad organizada: "Permanencia operativa, estructura organizacional, práctica de negocios ilícitos, planificación de actividades, empleo de redes de protección, dinámica movilidad 


\section{Revista científica, arbitrada e indizada, bajo la modalidad electrónica.}

internacional, requerimiento de fuentes de apoyo, fin lucrativo y concierto de alianzas estratégicas o tácticas”. (p.61).

\section{Elementos identificadores del crimen organizado}

Mejías (2014), citando a World Drug Report (2013), indica que en este caso, aun cuando también hay es necesario tener muy presente la diversidad regional, lo cierto es que, en mayor o menor medida, la violencia es un problema generalizado y en particular para países como México, Colombia, Venezuela, subregiones de Centroamérica. No obstante, incluso Brasil pese a ser líder regional y aspirante a convertirse en internacional, soporta el azote de esta violencia criminal. El motivo se encuentra en la implantación del crimen organizado, organización de maras o pandillas juveniles, vinculadas o no a este flagelo, al mismo crecimiento de la delincuencia común. De todas las actividades ilícitas en desarrollo por estos grupos criminales, el narcotráfico se constituye en el negocio por excelencia.

De estas aseveraciones no escapa América Latina, conocida por ser productora de cocaína y abastecer a los Estados Unidos, vecino y primer consumidor mundial de drogas. Aun cuando debe reconocerse el rentable negocio que supone la demanda europea. Además de región productora distribuidora, en los últimos años ha empezado a ser consumidora, enredando aún más la situación.

Sustenta de igual manera, Mejías (2014), que lo descrito infiere poner de manifiesto hasta donde los extraordinarios índices de violencia e inseguridad de la región son originados por la debilidad de instituciones estatales, en general, y de las relacionadas con la seguridad, en particular. Considerando es un problema presente en todos los países de la región. Efectivamente, aun cuando no hay Estados fallidos en América Latina, la impotencia de los mismos, puede hacer de sus naciones un campo más propicio, además de atractivo para la implantación, así como desarrollo, de actividades ilícitas.

Por ello, en el contexto de un Estado débil, caracterizado por la falta de institucionalidad y la excepcionalidad ante la ley, el crimen organizado, mediante la corrupción, tiene más posibilidades de influir en las decisiones estatales y de lograr la protección de sus autoridades. 


\section{Revista científica, arbitrada e indizada, bajo la modalidad electrónica.}

Finalmente, en este punto, el referido autor, asegura que, la presencia de mecanismos de rendición de cuentas, la aplicación de la ley, al igual la implementación de altos niveles de transparencia, contribuyen a disminuir posibilidades de desarrollar nexos de cooperación entre el crimen organizado y algunos representantes estatales. Situación muy común, en Estados débiles con democracias no consolidadas, en donde la carencia de efectividad de los mecanismos de control, además de transparencia, así como la falta de aplicación del imperio de ley para todos los ciudadanos, proporcionando espacios para las relaciones clientelares, personales, empleadas dispuestas a lograr, a través de la corrupción, la protección estatal.

\section{Tipologías de las estructuras criminales}

Según Páucar (2016), la clasificación más contemporánea corresponde al año 2002, la misma está relacionada precisamente con una investigación del Centro para la Prevención Internacional del Delito (CICP) del Instituto Interregional de Investigación de la Delincuencia y la Justicia de las Naciones Unidas (Unicri). Asumiendo, en esta oportunidad, datos de cuarenta agrupaciones criminales de dieciséis países, así como un método que asumió diez principales características: estructura, tamaño, violencia, identidad social, además de étnica, actividades, operaciones transfronterizas, corrupción, influencia política, mezcolanza entre actividades lícitas e ilícitas, y cooperación con otros grupos de organizaciones criminales, se instauran cinco tipologías, considerando la estructura el factor central de las demás.

\section{Tipología 1. Jerarquía estándar}

Conocida como estructura piramidal, simboliza la estructura más rígida y común entre los grupos de criminalidad organizada (Beare 2012). Cuenta con un comando o liderazgo unido, con el cual crea una jerarquía vertical con funciones definidas asignadas a sus integrantes (Prado 2006). Según Páucar (2016), en esta tipología las organizaciones criminales tienen un nombre que las identifica, comprende a aquellas dedicadas al sicariato, secuestro, robo, además de la usurpación agravada, extorsión, tráfico ilegal de armas, y el tráfico ilícito de drogas, modalidades delictivas contempladas en el artículo 3 de la Ley 30077-Ley contra el Crimen Organizado. 


\section{Revista científica, arbitrada e indizada, bajo la modalidad electrónica.}

\section{Tipología 2. Jerarquía regional}

Conserva como particularidad de la tipología un liderazgo concentrado en un solo sujeto. Idéntico a la tipología anterior, su característica esencial es el liderazgo único. Sin embargo, mantiene una jerarquía rígida y definida contando con un orden central, del cual se distribuyen organizaciones regionales con un significativo nivel de autonomía operativa (Beare 2012). En consecuencia, Páucar (2016), reseña como ejemplo las organizaciones criminales dedicadas al contrabando en zonas fronterizas, fundamentándose en la premisa que sus puntos de acopio y distribución, difieren según el tipo de mercancía, los cuales se mantienen como pequeños centros de operaciones a fin de garantizar objetivos formulados.

\section{Tipología 3. Agrupación jerárquica}

Los estudiosos de la temática señalan que esta tipología identifica una estructura corporativa la cual reúne varios grupos criminales (López 2015). Por lo tanto, la orientación de la agrupación subordinada es conferida a un núcleo de representantes de cada uno de los grupos integrados (Beare 2012). Páucar (2016), asegura no identificar en el país a un tipo en particular, señalando como ejemplo, solo el caso de la agrupación de las cinco familias de la mafia italoamericana: los Bonano, los Colombo, los Gambino, los Genovese y los Lucchese, quienes actuaban representadas por un comité.

\section{Tipología 4. Grupo central}

Es la tipología distintiva de las estructuras flexibles (López 2015). Conforma un modelo de estructura habitual en la organización criminal moderna (Prado 2006). Asumiendo como rasgo primordial la elasticidad, adaptabilidad, especialmente, la intercambiabilidad de sus miembros y funciones por dos criterios elementales. La primera direccionada al número reducido de integrantes (De la Corte y Giménez-Salinas 2010), la segunda se relaciona con la especialización que requiere sus planes delictivos. Como ejemplo, Páucar (2016), presenta las $\mathrm{O} / \mathrm{C}$ dedicadas al lavado de activos, las cuales cuentan con abogados, contadores, administradores, banqueros o notarios para crear empresas de fachada, además aquellas dedicadas a cometer delitos informáticos, integradas por personas con conocimientos de ingeniería de sistemas e informática conocidos como hackers. 


\section{Revista científica, arbitrada e indizada, bajo la modalidad electrónica.}

\section{Tipología 5. Red criminal}

Designada red criminal por su apariencia a una red artesanal; este lugar, los puntos nodales son los hombres clave, quienes se encargan de dar cimiento a la estructura de la red, al mismo tiempo, se enlazan mediante sus propios lazos, los cuales son las conexiones.

No cuentan con muchos integrantes, pero sí de cómplices temporales (De la Corte y Giménez-Salinas 2010). De acuerdo con Prado (2006), la red criminal sería una organización flexible, desarrolladora de actividades profesionales e intercambiables. Por ello, es la estructura criminal de diseño más compleja. El tamaño, así como actividades son variables. Para Páucar (2016), uno de los ejemplos más claros son las redes de corrupción originadas en la década de 1990, operando con grupos instituidos en los diversos niveles del poder político, instituciones del Estado, en algunos medios de comunicación, en las cuales existía un individuo clave encargado de diseñar la red criminal de corrupción tanto pública como privada, a los fines de operar el sistema, gestionando toda la actividad.

\section{Redes sociales}

El proceso de globalización, según Cajiao et al., (2018), así como los avances tecnológicos en las comunicaciones, la alta frecuencia de movimientos transfronterizos de personas y de bienes, ocasionaron la metamorfosis de las estructuras del crimen organizado. Citando a (Linares, 2008), señalan que ya no se requieren grandes organizaciones jerárquicas como los cárteles del narcotráfico colombiano o las mafias sicilianas. Por el contrario, el crimen organizado se estructura a través de redes sociales, fluidas, flexibles, con un alto grado de adaptación, rememorando el comportamiento de las corporaciones transnacionales cuando operan a través de redes y nodos funcionando a nivel global, tal como ha sido planteado por (Williams, 2008; Linares, 2008; Pérez, 2007).

Citando a Williams (2008), sostienen los referidos autores, el funcionamiento del crimen transnacional en redes, proporciona las interacciones entre estructuras, permite adaptarse con mayor facilidad a la acción de las autoridades y además las hace adaptables a los cambios que puedan originarse producto de su gestión. 


\section{Revista científica, arbitrada e indizada, bajo la modalidad electrónica.}

\section{Acciones diferenciadoras del crimen organizado}

A los fines de establecer cuales acciones se tornan diferentes en cada uno de los países objeto de estudio, se revisa lo expuesto por:

Seguridad Nacional (2019), para quienes la estrategia tiene entre las acciones, desarticulación de las estructuras criminales; reducción de sus actuaciones; dificultar la implantación de nuevos grupos; reducir este tipo de impacto ante la sociedad; además de contrarrestar la creciente vinculación entre terrorismo y crimen organizado.

Para ello, las acciones diferenciadoras se visualizan a partir de la estrategia, la cual queda estructurada en torno a diez ejes de actuación, siete troncales y tres trasversales, orientados a proporcionar una respuesta global a estos fenómenos complejos, con tantas dimensiones, así como singularidades: el crimen organizado y la delincuencia grave. Considerando que la estrategia marca también para cada uno de los ejes, líneas de acción, herramientas de utilidad para los operadores de seguridad, indistintamente donde se encuentren.

\section{Prevención y anticipación}

De igual forma, Seguridad Nacional (2019), ofrece las alternativas de planes estratégicos que buscan promover la generación de inteligencia como vehículo de anticipación contra estos fenómenos. Asegurando herramientas que coadyuven a erradicar o minimizar las nuevas tendencias de la realidad criminal, mediante el empleo de la inteligencia. Para lo cual resulta esencial el conocimiento sobre la situación, así como la evolución.

\section{Factores que favorecen la violencia articulando al crimen organizado}

En este aparte, Cajiao et al., (2018), señala la posibilidad de identificar por lo menos cuatro factores que inciden en el establecimiento de las estructuras del crimen organizado, las cuales facilitan la construcción de las redes criminales logísticas determinantes de las rutas para el tráfico internacional de drogas: (1) las condiciones sociopolíticas y económicas; (2) la incapacidad estatal para proveer justicia y debilidad en los sistemas judiciales; (3) la migración; (4) la utilización de estructuras criminales preexistentes.

De inmediato se describen cada una de ellas, en los siguientes términos: 


\section{Revista científica, arbitrada e indizada, bajo la modalidad electrónica.}

\section{Las condiciones sociopolíticas y económicas}

Las organizaciones criminales buscan aprovecharse de las vulnerabilidades de los Estados. En este sentido, para Maselli (2011), la falta de capacidad estatal para proveer servicios de calidad, así como la existencia de un gran sector económico informal de amplios sectores de la sociedad sin acceso a la economía formal, son grietas en la cohesión social y política ocupadas, además de magnificadas por el Crimen Organizado. En el caso colombiano, estas brechas sociales son aprovechadas por las organizaciones criminales para insertarse en las sociedades, legitimando su accionar criminal dentro de las mismas, ya que se convierten en proveedores de bienes y servicios. De igual forma, sucede en países con cierta vulnerabilidad, entre ellos Brasil, Guatemala y Venezuela.

\section{La incapacidad estatal para proveer justicia y debilidad en los sistemas judiciales}

Para algunos de los autores, la impunidad o la incapacidad de los Estados y sistemas judiciales para castigar o hacer cumplir las leyes, constituyen factores determinantes que estimulan el establecimiento de estructuras, así como redes criminales. En este caso, la impunidad, determinada por la corrupción de los sistemas de justicia, además la inoperancia de los mismos, facilitan el funcionamiento de las redes criminales y desincentivan el cumplimiento de las leyes. (AMERIPOL, 2013).

\section{RESULTADOS}

Ramos y Álvarez (2019), exponen que en América Latina, este flagelo está perturbando a las democracias, estimuladas por el efecto en ellas, de casos evidentes de utilización indebida de los recursos públicos y de autoridad soberana para alcanzar fines privados.

De igual forma, para los citados autores, la corrupción se ha convertido en uno de los factores más incidentes en la desconfianza generada en las instituciones democráticas latinoamericanas.

Mejías (2014), expone que precisamente a través de la corrupción, el crimen organizado consigue la protección y complicidad del Estado. Esta impunidad es la que le permite tener control del territorio, así como protegerse de organizaciones rivales o del propio Estado. 


\section{Revista científica, arbitrada e indizada, bajo la modalidad electrónica.}

Al examinar lo expuesto por diferentes autores, resulta evidente la estrecha relación existente entre la corrupción y el crimen organizado, porque precisamente es el cultivo para germinar acciones delictuales que traspasan fronteras. Contribuyendo al deterioro de las estructuras gubernamentales e institucionales. Por ello, para conocer la situación actual de la violencia como fenómeno transnacional, es necesario revisar los cuatros elementos con mayor incidencia en esta problemática.

Como puede evidenciarse en el contenido expuesto al realizar la presentación e interpretación de resultados, la situación actual de la violencia como fenómeno transnacional en los países contextualizados, refleja una realidad dominada por actividades ilícitas que traspasan las fronteras, tales como la corrupción, el narcotráfico, la extorsión y el secuestro.

Es evidente a partir de los resultados arrojados, que los elementos identificadores del crimen organizado en cada uno de los países objeto de análisis, se encuentran enmarcados en dos grandes elementos, la estructura de la acción delictiva que deriva de las respectivas tipologías y las redes sociales implementadas para cometer actividades ilícitas.

\section{CONCLUSIONES}

Los resultados derivados son indicativos de que la acción delictiva comprende un accionar de violencia en todas sus manifestaciones, contribuyendo a fomentar actividades al margen de la ley que trascienden fronteras geográficas.

La violencia en sí misma, propicia un accionar al margen de la ley, exaltando la perpetración de conductas ilícitas que conllevan al deterioro de estructuras familiares, sociales, institucionales, entre otros. En este sentido, ha quedado evidente que en los países objeto de estudio, los eventos sociopolíticos acaecidos, así como la desestabilización económica acontecida han dado lugar a una violencia desmedida en contra de órdenes gubernamentales, democráticamente instaurados, llevando a un clima de desconfianza al acrecentarse la inseguridad de personas y bienes materiales. 


\section{Revista científica, arbitrada e indizada, bajo la modalidad electrónica.}

De igual forma, el trabajo socializado a través de este artículo, arroja como resultado que en los cuatro países examinados, la violencia generada producto de factores económicos, políticos y sociales, así como la ingorbenabilidad en estos Estados, se encuentra vinculada directamente con la generación del crimen organizado, ya que es evidente su afianzamiento en todas las modalidades, trayendo consigo deterioro de las instituciones públicas garantizadoras del bienestar social, debido a la impunidad ante las acciones delictivas.

Al igual que en otros países del mundo, este flagelo no solo afecta la institucionalidad del estado al hacerlo ingobernable, sino la seguridad de las personas, quienes se sienten constantemente amenazadas en su integridad física. Adicionalmente, estimula la corrupción, favoreciendo otras acciones delictivas como el secuestro y la extorsión.

\section{REFERENCIAS BIBLIOGRÁFICAS}

AMERIPOL (2013). Análisis situacional del narcotráfico "una perspectiva policial": Bolivia, Brasil, Colombia, Ecuador, Panamá y Perú. Fundación Internacional y para Iberoamérica de Administración y Políticas Públicas.

Basset, Y; Tahar, M; Aguilar, M. (2015). Procesos democráticos en contextos de violencia: México y Colombia. Editorial Universidad del Rosario. Bogotá. Colombia.

Beare, M. E. (2012). Enciclopedia of Trasnational Crime and Justice. Washington D.C.: Sage.

Bernall, J. (2015). Proceso Penal. Colombia. Colombia: Universidad Externado de Colombia.

Caballero, C; Pizano, D. (2014). Punto de inflexión: Decisiones que rescataron el futuro de Colombia. Ediciones Uniandes. Colombia.

Cabrera, E. (2017). La trata de seres humanos con fines de explotación sexual: la detección e identificación de las víctimas. (Tesis doctoral. Universidad Camilo José Cela, Madrid).

Cajiao, A; González, P; Pardo, D; Zapata, O. (2018). Una aproximación al crimen transnacional organizado: redes de narcotráfico Colombia-España. Fundación Ideas para la paz. Documento de trabajo. Disponible en: http://cdn.ideaspaz.org/media/website/document/5ac3f4c9d69a1.pdf

Capera Figueroa, J; Nañez Rodríguez, J. (2017). Una perspectiva crítica sobre la violencia en Colombia en épocas del postconflicto. Utopía y Praxis Latinoamericana, [S.1.], v. 22, n. 78, p. 153-162, jul. ISSN 2477-9555. Disponible en: <http://produccioncientificaluz.org/index.php/utopia/article/view/22645/22451 >. Fecha de acceso: 10 mar. 2020 


\section{Revista científica, arbitrada e indizada, bajo la modalidad electrónica.}

Carrara, F. (2014).Programa de Derecho Criminal. Parte Especial. Bogotá. Colombia: Editorial Temis.

Código Penal Venezolano (2011). Gaceta Oficial de la República Bolivariana de Venezuela $\mathrm{N}^{\circ}$ 39.818. Diciembre 12, 2011.Caracas. Venezuela.

Chihaia, M. (2019). La violencia como marco interpretativo de la investigación literaria. Publicado por Narr Francke Attempto Verlag. Disponible en: https://books.google.co.ve/books?id=BJOtDwAAQBAJ\&pg=PT34\&dq=\%22\%22artic ulos+sobre+la+violencia+en+am\%C3\%A9rica+latina+2019\%22\%22\&hl=es419\&sa=X\&ved=0ahUKEwjUsbqdiNPoAhXQW80KHWHZALsQ6AEINjAC\#v=one page \&q $=\% 22 \% 22$ articulos\%20sobre\%20la\%20violencia\%20en\%20am\%C3\%A9rica $\%$ 20latina\%202019\%22\%22\&f=false

De la Corte, L., y Giménez-Salinas, A. (2010). Crimen.Org. Evolución y claves de la delincuencia organizada. Barcelona: Ariel.

González Luna, F. (2013). Espacialización de la violencia en las ciudades latinoamericanas: una aproximación teórica. Cuadernos de Geografía: Revista Colombiana de Geografía, 22(1), 169-186.

Maselli, C. (2011). Seguridad y crimen organizado transnacional: una propuesta de acción para Centroamérica, Fundación Konrad Adenauer - Red Centroamericana de Centros de Pensamiento e Incidencia, Guatemala.

Mejías, S. (2014). Estado y crimen organizado en América Latina: Posibles relaciones y complicidades. Revista Política y Estrategia No 124: 73-107.

Mesa Peinado, M. (2018). Globalización y transnacionalización de la violencia en Centroamérica, en los países del Triángulo Norte. Tesis Doctoral Universidad Complutense de Madrid. España. Disponible en: https://dialnet.unirioja.es/servlet/tesis?codigo $=152734$

Universidad Yacambu (2007). Normas UNY (2007). Disponible en: http://www.uny.edu.ve Observatorio venezolano de violencia. (2020). Balance anual de los homicidios, InSight Crime los homicidios 2019. Disponible en: https://observatoriodeviolencia.org.ve/news/balance-de-insight-crime-de-loshomicidios-en-2019/

Páucar, M. E. (2016). El delito de organización criminal. Lima: Ideas Solución Editorial.

Pelekais, C; Pertuz, F; Pelekais, E (2016) Hacia una cultura de investigación cualitativa. Ediciones Astro Data S.A Maracaibo. Venezuela.

Pelekais, C; El Kadi, O; Seijo, C; Neuman, N (2015). El ABC de la Investigación. Guía Didáctica. Ediciones Astro Data S.A. Maracaibo. Venezuela.

Prado, V. R. (2013). Criminalidad organizada y lavado de activos. Lima: Idemsa.

Prado, V. R. (2006). Criminalidad organizada. Lima: Idemsa. 


\section{Revista científica, arbitrada e indizada, bajo la modalidad electrónica.}

Sampó, C. (2018). Brasil: la re-significación de la violencia como resultado del avance de organizaciones criminales. Revista de estudios en seguridad internacional. Vol. 4, No. 1, pp. 127-146. DOI: http://dx.doi.org/10.18847/1.7.8. Disponible en: http://www.seguridadinternacional.es/revista/?q=content/brasil-la-resignificaci\%C3\%B3n-de-la-violencia-como-resultado-del-avance-de-organizaciones

Seguridad Nacional (2019). Estrategia nacional contra el crimen organizado y la delincuencia grave. Disponible en: https://www.dsn.gob.es/es/actualidad/salaprensa/estrategia-nacional-contra-crimen-organizado-delincuencia-grave

Solis, J; Cerna, S. (2014). Inseguridad y violencia en América Latina: una mirada crítica a sus causas estructurales. Anuari del conflicte social.

Ramos, M; Álvarez, F. (2019). El control de la corrupción en América Latina: agenda política, judicialización e internacionalización de la lucha contra la corrupción. Documentos de trabajo Fundación Carolina. Agenda 2030. Disponible en: https://www.fundacioncarolina.es/wp-content/uploads/2019/07/DT_FC_11.pdf 